\title{
Comparison of Effect of Metformin Versus Combination of Folic Acid/Myo-inositol in Infertile Women with Poly Cystic Ovary Syndrome Undergoing In Vitro Fertilization: A Randomized Clinical Trial
}

\author{
Fereshteh Rastegar ${ }^{1, *}$, Zahra Rezaee ${ }^{2}$, Nafiseh Saedi' ${ }^{2}$, Reza Memari ${ }^{3}$, Mahnaz Tajpour ${ }^{4}$
}

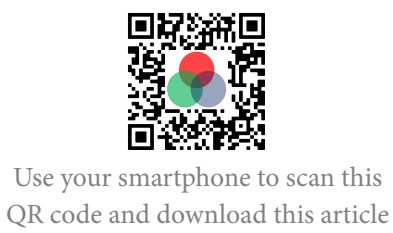

${ }^{1}$ Department of Obstetrics and Gynecology, Modares and 17 Shahrivar hospital, Saveh University of Medical Sciences, Saveh, Iran

${ }^{2}$ Department of Obstetrics and Gynecology, Yas hospital, Tehran University of Medical Sciences, Tehran, Iran

${ }^{3}$ Management operation and Technology, Flinders University, Australia

${ }^{4}$ Rask Hospital, Iranshahr University of Medical Sciences, Iranshahr, Iran

\section{Correspondence}

Fereshteh Rastegar, Department of Obstetrics and Gynecology, Modares and 17 Shahrivar hospital, Saveh

University of Medical Sciences, Saveh, Iran

Email: fereshteh.rastegar61@gmail.com

\section{History}

- Received: Aug 30, 2021

- Accepted: Nov 21, 2021

- Published: Dec 30, 2021

DOI : 10.15419/bmrat.v8i12.710

\section{Check for updates}

\section{Copyright}

(๑) Biomedpress. This is an openaccess article distributed under the terms of the Creative Commons Attribution 4.0 International license.

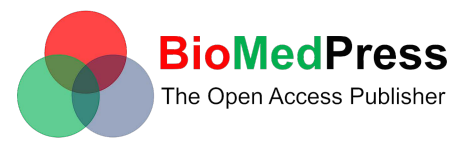

\begin{abstract}
Background: Previous studies have reported contradictory results regarding the effectiveness of metformin and myo-inositol on improving reproductive outcomes in patients with polycystic ovary syndrome (PCOS). Therefore, this study aimed to compare the effectiveness of these two drugs on improving reproductive outcomes of PCOS patients undergoing in vitro fertilization (IVF) treatment. Methods: This double-blind randomized clinical trial study was conducted on 140 infertile women with PCOS who were scheduled to undergo IVF in Yas Hospital, Tehran, Iran in 2020. The patients were randomly divided into two groups, including metformin and combination of myo-inositol and folic acid treatment groups. Reproductive outcomes were compared between the two groups. Results: The mean age of the patients was $30.66 \pm 5.7$ years ( $30.51 \pm 5.88$ in metformin treatment group and $30.9 \pm 5.53$ in myo-inositol group, $P=0.68$ ). There was no significant difference between the two groups regarding the reproductive outcomes, including pregnancy rate, change in BMI, number and quality of oocytes, number and quality of embryos, follicle number, follicle size, ectopic pregnancy rate, and abortion rate $(P>0.05)$. Conclusion: There is no significant difference in the effect of metformin and myo-inositol on improving reproductive outcomes. Therefore, due to the lack of significant differences in the effectiveness of these two drugs and considering their availability and cost, one of them can be prescribed for the patients.

Key words: PCOS, metformin, Myo-Inositol, Infertility, Randomized clinical trial
\end{abstract}

\section{INTRODUCTION}

Infertility is considered an inability to conceive, despite having regular unprotected intercourse for a year or inability to carry a pregnancy to full term ${ }^{1}$. In general, the prevalence of infertility in young couples is estimated to be in the range $10-15 \%{ }^{2}$. Ovulation problems are the most common causes of infertility in women. In $80 \%$ of cases, infertility occurs due to certain conditions such as endometriosis or polycystic ovary syndrome $(\mathrm{PCOS})^{3}$. PCOS is an endocrine disorder that affects $6-15 \%$ of women in reproductive age in different geographical areas ${ }^{4}$.

Insulin resistance is the pathophysiological basis of PCOS and some women with this syndrome suffer from metabolic problems. There is evidence of pregnancy in women with PCOS who have undergone assisted reproductive techniques with hyperandrogenism or insulin resistance while taking drugs such as metformin or inositol ${ }^{2}$. Metformin, as the most common insulin sensitizer, is used for the treatment of PCOS worldwide. Metformin, as a biguanide insulin-lowering agent, has been widely studied in the management of PCOS. Two previously published systematic reviews reported that metformin improves the reproductive function of women with PCOS ${ }^{5,6}$. Metformin seemed to diminish oestradiol levels and reduce the risk of over stimulation ${ }^{7}$. Moreover, metformin improves the outcomes of ovulation induction therapies when combined with clomiphene and gonadotropin ${ }^{6}$.

However, its use may be limited due to its adverse effects on the gastrointestinal tract ${ }^{8,9}$. Due to several complications of metformin such as nausea and vomiting as well as poor compliance to this drug, there is a growing interest in physicians to use alternative drugs. Moreover, evidence-based studies showed its effects on myo-inositol as a food supplement in the treatment of metabolic complaints of reproduction such as PCOS in women, whereby it improves the quality of oocytes and embryo ${ }^{10,11}$. Previous studies indicated a direct correlation between concentration of myo-inositol in the follicular fluid and high oocyte quality. These studies have reported that myo-inositol

Cite this article: Rastegar F, Rezaee Z, Saedi N, Memari R, Tajpour M. Comparison of Effect of Metformin Versus Combination of Folic Acid/Myo-inositol in Infertile Women with Poly Cystic Ovary Syndrome Undergoing In Vitro Fertilization: A Randomized Clinical Trial. Biomed. Res. Ther., 2021; 8(12):4734-4739. 
is able to improve oocyte quality ${ }^{\mathbf{1 2}-15}$. However, contradictory results have been reported regarding their effects on improving reproductive outcomes. Hence, the aim of this study was to compare the effectiveness of metformin and myo-inositol in improving reproductive outcomes, including fertilization rate, follicle size, embryo count, embryo quality, follicle count, abortion rate, ectopic pregnancy rate (EP) rate, and OHSS rate for women with PCOS undergoing in vitro fertilization (IVF).

\section{METHODS}

\section{Study design}

This double-blind randomized clinical trial was conducted on 140 infertile women with PCOS referred to the infertility clinic of Yas Hospital from February to June 2020, Tehran, Iran. The patients were scheduled to undergo IVF and had no other cause of infertility. The patients were randomly divided into two groups. The standard treatment group received $500 \mathrm{mg}$ of metformin three times a day, and the intervention group received a combination of $2000 \mathrm{mg} / 200 \mu \mathrm{g}$ myo-inositol along with follicle acid twice a day. Balanced block randomization was used to classify the subjects into two groups, and finally 70 patients were assigned to each group.

\section{Eligibility criteria}

We included patients with the following criteria: (1) Patients aged 18 - 40 years, (2) first IVF treatment, and (3) patency of fallopian tubes. The exclusion criteria were infertility due to male infertility factor, having a history of ovarian manipulation, and smoking. All of the participants signed a written informed consent form.

\section{Study procedure}

After obtaining the informed consent form in the infertility clinic of Yas Hospital, the included patients were randomly divided into two groups. Demographic information, including age, weight, height, body mass index, duration of infertility, type of infertility, history of abortion, and EP were gathered from their medical records or through interview. Random allocation to groups was done by an infertility resident, starting from three months before IVF cycle until ovum puncture. The size and count of follicles was recorded by a trans-vaginal ultrasound. The IVF was performed in the IVF operating room of the Yas Hospital. The patients were followed for pregnancy duration by beta HCG and ultrasound, as well as for abortion and EP. OHSS were carefully monitored for events during the cycle. In this study, the researchers and the statistical analyzer were blinded to the treatment groups (double-blind).

\section{Outcomes}

In this study, the primary outcome was the pregnancy rate, and the secondary outcomes included BMI change, fertilization rate, embryo count, embryo quality, follicle count, follicle size, abortion rate, EP rate and OHSS rate. Pregnancy was defined as a positive test at two weeks from ET. Oocyte maturation was defined by the percentage of metaphase II (MII) oocytes. Embryos were assessed according to the ESHRE criteria.

\section{Data analysis}

Data were analyzed using SPSS software (version 24.0, SPSS Inc., Chicago, IL). The qualitative data were presented in frequency and percentage, and the quantitative variables were presented as mean $\pm \mathrm{SD}$. The normality of data was assessed with the KolmogorovSmirnov test. The continuous variables of the two groups were compared by Student's t-test, and the categorical variables were compared using the chisquared test. The p-value of less than 0.05 was considered the significance level.

\section{Ethical considerations}

Ethical approval for the study was obtained from the Ethics Committee of Tehran University of Medical Sciences, Tehran, Iran. This clinical trial was registered in the Iranian Registry of Clinical Trials (IRCT) with the IRCT ID: IRCT20201219049758N1 (http:// www.irct.ir).

\section{RESULTS}

In the present study, 166 infertile women with PCOS who were scheduled to undergo IVF were screened. Of them, 19 patients did not meet the inclusion criteria and 7 patients were unwilling to participate in the present study. Therefore, 140 patients were evaluated and classified into two groups. These patients were randomly assigned into two groups (70 patients in the metformin treatment group and 70 patients in the myo-inositol treatment group). There was no loss of participants during the study, and therefore all of the included patients were entered into the final analysis (Figure 1).

In this study, the parameters of Agrawal et al.s study were considered, and the level of alpha $=0.05$ and power $=0.9$ for sample size calculation obtained 70 cases for each arm of the study ${ }^{16}$. 


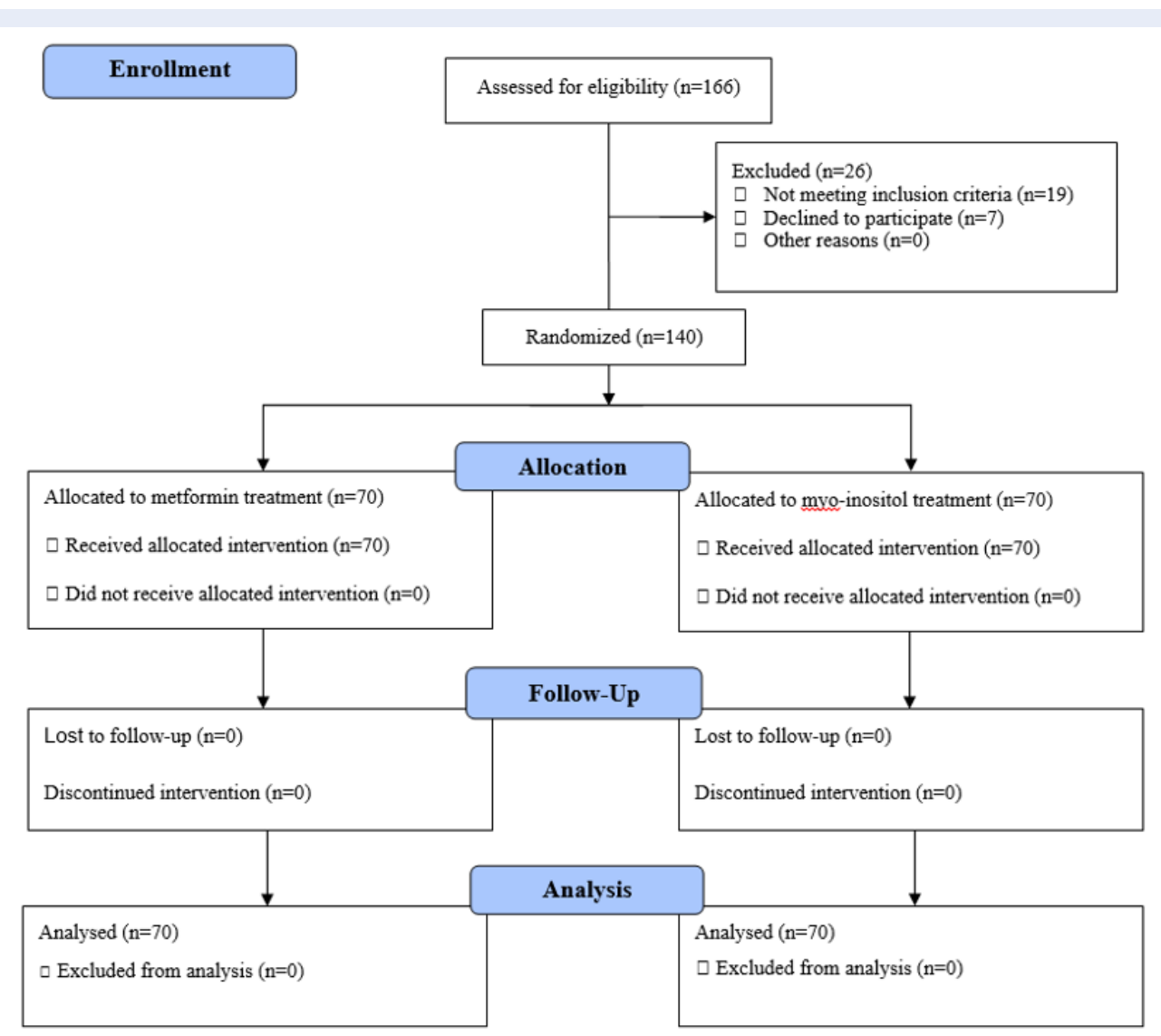

Figure 1: Flowchart of allocation of patients to the studied groups.

The mean age of the patients was $30.66 \pm 5.7$ years $(30.51 \pm 5.88$ in the metformin group and $30.9 \pm 5.53$ in the myo-inositol group, $\mathrm{P}=0.68$ ). Moreover, there was no significant difference between the two groups in terms of duration of infertility, BMI, parity, gravity, and history of abortion $(\mathrm{P}>0.05)$ (Table 1).

In Table 2, the reproductive outcomes were compared between the two investigated groups. As shown here, the two groups were homogenous in terms of pregnancy rate, change in BMI, number and quality of oocytes, number and quality of embryos, follicle number, follicle size, ectopic pregnancy rate, and abortion rate $(\mathrm{P}>0.05)$.

\section{DISCUSSION}

In this study, the effects of metformin and myoinositol administration on improving reproductive outcomes were compared in women with PCOS undergoing IVF. The results demonstrated that there was no significant difference between the two groups receiving these two drugs, in terms of pregnancy rate, change in BMI, number and quality of oocytes, number and quality of embryos, follicle number, ectopic pregnancy rate, abortion rate, and follicle size.

Consistent with our results, a previous study compared the effects of metformin and myo-inositol on PCOS patients, and it was found that there was no significant difference in BMI between the two treatment groups ${ }^{10}$. In Nas et al's study, regarding the comparison of therapeutic efficacy of myo-inositol versus metformin treatment in insulin resistance patients, the BMI was moderate, but there was no significant difference between the two treatment methods ${ }^{17}$. Nehra et al. reported a decrease in BMI, which is consistent with the results of the present study ${ }^{\mathbf{1 8}}$. Moreover, Gerli et al. showed that body weight decreased significantly in the patients treated with myoinositol, and they concluded that myo-inositol treatment might possibly reduce the risk of cardiovascular diseases in PCOS women ${ }^{19}$.

No significant difference was observed between the two treatment groups in terms of pregnancy rate and ovulation activity, while Raffone et al. showed a 50\% 
Table 1: Comparison of baseline characteristics between two groups

\begin{tabular}{lcccc}
\hline Variable & & Metformin group & Myo-inositol group & P-value \\
\hline Gravity & 0 & $40(48.78)$ & $42(51.22)$ & 0.57 \\
& 1 & $20(47.62)$ & $22(52.38)$ & \\
\hline Parity & 2 & $10(62.50)$ & $6(37.50)$ & 0.93 \\
& 0 & $47(48.96)$ & $49(51.04)$ & $18(47.37)$ \\
\\
History of abortion & 1 & $20(52.63)$ & $3(50.0)$ & 0.64 \\
& 2 & $3(50.0)$ & $60(50.85)$ & \\
History of Ectopic & No & $58(49.15)$ & $10(45.45)$ & 0.57 \\
pregnancy & Yes & $12(54.55)$ & $64(50.79)$ & $6(42.86)$ \\
\hline Age (year) & No & $62(49.21)$ & $30.9 \pm 5.53$ & 0.68 \\
Duration of infertility (year) & Yes & $8(57.14)$ & $6.30 \pm 4.20$ & 0.97 \\
BMI (Kg/m ${ }^{2}$ ) & & $30.51 \pm 5.88$ & $23.35 \pm 4.83$ & 0.39 \\
\hline
\end{tabular}

Table 2: Reproductive outcomes in the two treatment groups

\begin{tabular}{lcccc}
\hline Variable & & Metformin group & Myo-inositol group & P-Value \\
\hline Pregnancy & Yes & $28(40.00)$ & $29(41.43)$ & 0.86 \\
Ectopic pregnancy & No & $42(60.00)$ & $41(58.57)$ & \\
& Yes & $1(1.43)$ & $2(2.86)$ & 0.56 \\
Abortion & No & $69(98.57)$ & $68(97.14)$ & 0.75 \\
& Yes & $5(7.14)$ & $6(8.57)$ & \\
Change in BMI $\left(\mathrm{Kg} / \mathrm{m}^{2}\right)$ & No & $65(92.86)$ & $64(91.43)$ & 0.25 \\
Number of oocytes & & $1.66 \pm 0.48$ & $1.57 \pm 0.47$ & 0.84 \\
Oocyte quality & & $7.17 \pm 4.40$ & $1.99 \pm 1.87$ & 0.63 \\
& GV & $2.14 \pm 2.04$ & $2.84 \pm 2.59$ & 0.92 \\
Follicle size & M1 & $2.89 \pm 2.52$ & $2.1 \pm 2.43$ & 0.97 \\
\hline & M2 & $2.08 \pm 2.2$ & $4.23 \pm 3.42$ & 0.67
\end{tabular}


restore in spontaneous ovulation activity and occurrence of spontaneous pregnancy in 11 patients in the metformin group, whereas in the present study, myoinositol slightly improved pregnancy rate compared to the metformin group ${ }^{20}$.

Ovarian dysfunction was observed in $70-80 \%$ of the women with PCOS presenting with oligomenorrhoea or amenorrhea ${ }^{21}$. In Angik et al's study, after six months of treatment, $28.57 \%$ of patients in the myoinositol and $48 \%$ of patients in the metformin treatment group achieved regular cycles. Moreover, in this study, the ovarian volume and follicle count significantly decreased in the both groups ${ }^{22}$. However, no statistically significant difference was observed between the two groups.

Inositols act as secondary messenger molecules for various cellular signaling pathways, including insulin signal transduction, lipid metabolism, cell growth and differentiation, oocyte maturation, and fertility. It has been reported that Inositol plays an important role in oocyte maturation and fertilization through calcium signalling pathways. The level of myo-inositol in the blood and follicular fluids is positively related to the quality of the oocytes and the outcome of pregnancy in humans. The results of the previous studies indicated that administration of myo-inositol to women before hormonal stimulation in IVF cycles increases the quality of oocytes and embryos, and subsequently reduces the dose of $\mathrm{FSH}^{9,23}$.

However, the present study had some limitations including lack of investigation of the side effects of the two drugs and lack of enough time to evaluate the long-term effect of drugs. Finally, the small sample size of the study for assessing some reproductive outcomes can be considered as another limitation of the study.

\section{CONCLUSIONS}

In the present study, no significant difference was observed in the effect of metformin and myo-inositol regarding improvement of the reproductive outcomes. Therefore, due to the lack of significant differences in the effectiveness of these two drugs and considering their availability and cost, one of the two drugs can be prescribed for the eligible patients.

\section{ABBREVIATIONS}

EP: ectopic pregnancy, IVF: in vitro fertilization, OHSS: Ovarian hyperstimulation syndrome, PCOS: Polycystic ovary syndrome

\section{ACKNOWLEDGMENTS}

This study was supported by the Deputy of Research and Technology of Tehran University of Medical Sciences. We gratefully acknowledge the kind support of the participants for their precious collaboration in this study as well as the staff of the Yas Hospital.

\section{AUTHOR'S CONTRIBUTIONS}

FR, ZR, and MT developed the original idea and the protocol, abstracted, and prepared the manuscript. FR and RM participated in the study design and analyzed the data. FR, ZR, and MT contributed to the data gathering. All authors read and approved the final manuscript.

\section{FUNDING}

This study was funded by the Tehran University of Medical Sciences, Iran.

\section{AVAILABILITY OF DATA AND MATERIALS}

Data and materials used and/or analyzed during the current study are available from the corresponding author on reasonable request. The data that support the findings of the study are available from the corresponding author in SPSS form upon reasonable request.

\section{ETHICS APPROVAL AND CONSENT TO PARTICIPATE}

Institutional review board approval was obtained from the ethics committees of Tehran University of Medical Sciences. Written and informed consent was obtained from the patients.

\section{CONSENT FOR PUBLICATION}

Not applicable.

\section{COMPETING INTERESTS}

The authors declare that they have no competing interests.

\section{REFERENCES}

1. Coad J, Pedley K, Dunstall M. Anatomy and physiology for midwives e-book: Elsevier Health Sciences; 2019.

2. Naderpoor N, Shorakae S, de Courten B, Misso ML, Moran LJ, Teede HJ. Metformin and lifestyle modification in polycystic ovary syndrome: systematic review and meta-analysis. Human Reproduction Update. 2015;21(5):560-74. PMID: 26060208. Available from: 10.1093/humupd/dmv025.

3. Ried K, Stuart K. Efficacy of Traditional Chinese Herbal Medicine in the management of female infertility: a systematic review. Complementary Therapies in Medicine. 2011;19(6):319-31. PMID: 22036524. Available from: 10.1016/ j.ctim.2011.09.003.

4. Gao L, Mao Q, Cao J, Wang Y, Zhou X, Fan L. Effects of coenzyme $\mathrm{Q} 10$ on vascular endothelial function in humans: a meta-analysis of randomized controlled trials. Atherosclerosis. 2012;221(2):311-6. PMID: 22088605. Available from: 10.1016/j.atherosclerosis.2011.10.027. 
5. Tang T, Norman RJ, Balen AH, Lord JM. Insulin-sensitising drugs (metformin, troglitazone, rosiglitazone, pioglitazone, D-chiro-inositol) for polycystic ovary syndrome. Cochrane Database of Systematic Reviews. 2003;2:CD003053. Available from: https://doi.org/10.1002/14651858.CD003053.

6. Costello MF, Eden JA. A systematic review of the reproductive system effects of metformin in patients with polycystic ovary syndrome. Fertility and Sterility. 2003;79(1):1-13. PMID: 12524053. Available from: 10.1016/S0015-0282(02)04554- 5.

7. Tasdemir S, Ficicioglu C, Yalti S, Gurbuz B, Basaran T, Yildirim G. The effect of metformin treatment to ovarian response in cases with PCOS. Archives of Gynecology and Obstetrics. 2004;269(2):121-4. PMID: 12764624. Available from: 10.1007/s00404-002-0447-8.

8. Fulghesu AM, Romualdi D, Florio CD, Sanna S, Tagliaferri V, Gambineri A, et al. Is there a dose-response relationship of metformin treatment in patients with polycystic ovary syndrome? Results from a multicentric study. Human Reproduction (Oxford, England). 2012;27(10):3057-66. PMID: 22786777. Available from: 10.1093/humrep/des262.

9. Palomba S, Falbo A, Zullo F, Orio F. Evidence-based and potential benefits of metformin in the polycystic ovary syndrome: a comprehensive review. Endocrine Reviews. 2009;30(1):1-50. PMID: 19056992. Available from: 10.1210/er.2008-0030.

10. Facchinetti $F$, Orrù $B$, Grandi $G$, Unfer V. Short-term effects of metformin and myo-inositol in women with polycystic ovarian syndrome (PCOS): a meta-analysis of randomized clinical trials. Gynecological Endocrinology. 2019;35(3):198-206. PMID: 30614282. Available from: 10.1080/09513590.2018. 1540578.

11. Unfer V, Facchinetti $F$, Orrù $B$, Giordani $B$, Nestler J. Myoinositol effects in women with PCOS: a meta-analysis of randomized controlled trials. Endocrine Connections. 2017;6(8):647-58. PMID: 29042448. Available from: 10.1530/ EC-17-0243.

12. Raffone $E$, Rizzo P, Benedetto V. Insulin sensitiser agents alone and in co-treatment with r-FSH for ovulation induction in PCOS women. Gynecological Endocrinology. 2010;26(4):275-80. PMID: 20222840. Available from: 10.3109/ 09513590903366996.

13. Papaleo E, Unfer V, Baillargeon JP, Fusi F, Occhi F, Santis LD. Myo-inositol may improve oocyte quality in intracytoplasmic sperm injection cycles. A prospective, controlled, randomized trial. Fertility and Sterility. 2009;91(5):1750-4. PMID 18462730. Available from: 10.1016/j.fertnstert.2008.01.088.

14. Chiu TT, Rogers MS, Law EL, Briton-Jones CM, Cheung LP, Haines CJ. Follicular fluid and serum concentrations of myo-inositol in patients undergoing IVF: relationship with oocyte quality. Human Reproduction (Oxford, England). 2002;17(6):1591-6. PMID: 12042283. Available from: 10.1093/ humrep/17.6.1591.

15. Unfer V, Carlomagno G, Rizzo P, Raffone E, Roseff S. Myoinositol rather than D-chiro-inositol is able to improve oocyte quality in intracytoplasmic sperm injection cycles. A prospective, controlled, randomized trial. European Review for Medical and Pharmacological Sciences. 2011;15(4):452-7. PMID: 21608442.

16. Agrawal A, Mahey R, Kachhawa G, Khadgawat R, Vanamail $P$, Kriplani A. Comparison of metformin plus myoinositol vs metformin alone in PCOS women undergoing ovulation induction cycles: randomized controlled trial. Gynecological Endocrinology. 2019;35(6):511-4. PMID: 30614289. Available from: 10.1080/09513590.2018.1549656.

17. Nas K, Tuu L. A comparative study between myo-inositol and metformin in the treatment of insulin-resistant women. European Review for Medical and Pharmacological Sciences. 2017;21(2):77-82. PMID: 28724173.

18. Nehra J, Kaushal J, Singhal SR, Ghalaut V. A comparative study of efficacy and safety of myo-inositol versus metformin in polycystic ovarian syndrome in women. World Journal of Pharmacy and Pharmaceutical Sciences. 2016;5(5):884-96.

19. Gerli S, Mignosa M, Renzo GCD. Effects of inositol on ovarian function and metabolic factors in women with PCOS: a randomized double blind placebo-controlled trial. European Review for Medical and Pharmacological Sciences. 2003;7(6):151-9. PMID: 15206484.

20. Lobo RA, Carmina E. The importance of diagnosing the polycystic ovary syndrome. Annals of Internal Medicine. 2000;132(12):989-93. PMID: 10858183. Available from: 10 7326/0003-4819-132-12-200006200-00010.

21. Brassard M, AinMelk Y, Baillargeon JP. Basic infertility including polycystic ovary syndrome. The Medical Clinics of North America. 2008;92(5):1163-92. PMID: 18721657. Available from: 10.1016/j.mcna.2008.04.008.

22. Angik R, Jajoo SS, Hariharan C, Chimote A. A comparative study of metabolic and hormonal effects of myoinositol vs. metformin in women with polycystic ovary syndrome: a randomised controlled trial. International Journal of Reproduction, Contraception, Obstetrics and Gynecology. 2015;4(1):189-94. Available from: 10.5455/2320-1770. ijrcog20150234.

23. Carlomagno G, Unfer V. Inositol safety: clinical evidences. European Review for Medical and Pharmacological Sciences. 2011;15(8):931-6. PMID: 21845803. 
Ready to submit your manuscript? Choose Biomedpress and benefit from:

- Fast, convenient online submission

- Through peer-review by experienced researchers

- Rapid publication on acceptance

- Free of charge (without publication fees)

Learn more http://www.biomedpress.org/journals/
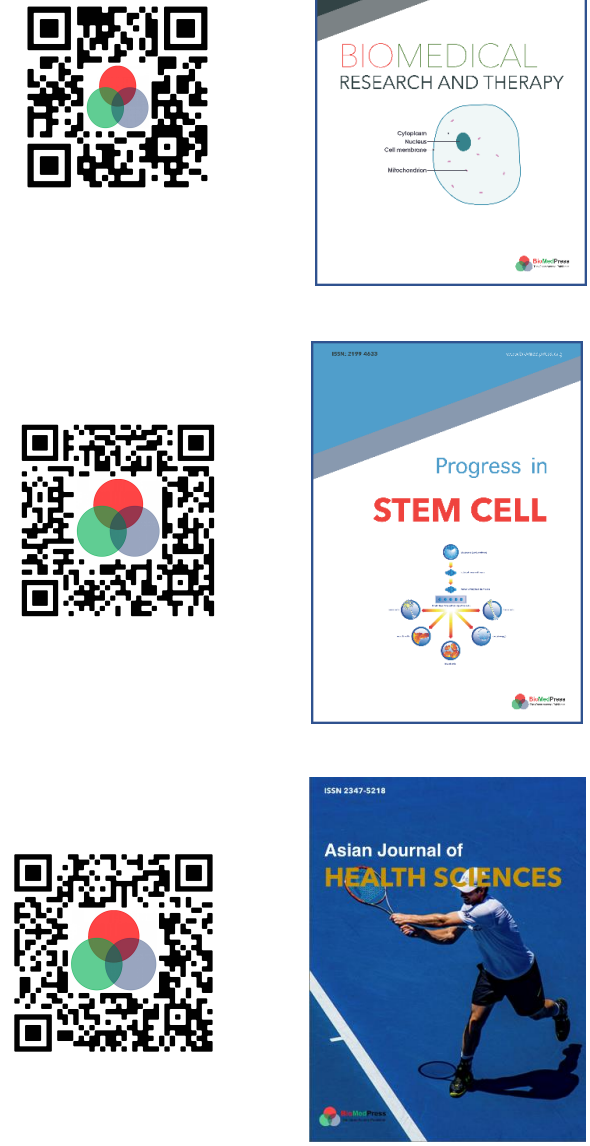

Asian Journal of Health Sciences

ISSN: 2347-5218

Indexed: Google Scholar

Acceptance Rate (2020): 72.89\%

Article Publishing Charge: Free

Submission to first editorial decision: 16.5 days

Biotechnological Research

ISSN: 2395-6763

Indexed: Google Scholar

Acceptance Rate (2020): $67.02 \%$

Article Publishing Charge: Free

Submission to first editorial decision: 28.5 days 